\title{
Clinicopathologic Features and Prognostic Role of CD3, CD8, and PD-1 positive Tumor-Infiltrating Lymphocytes and the Association with COX-2 Overexpression in Endometrial Carcinoma
}

\author{
Niusha Nobari $\mathbb{D}^{1}{ }^{1}$, Nakisa Niknejad ${ }^{2}$, Jalal Poorolajal ${ }^{3}$, Maryam Vaezi ${ }^{4}$, Fatemeh Nili $\mathbb{D}^{1}{ }^{1,}{ }^{*}$ and Issa \\ Jahanzad $^{1}$ \\ ${ }^{1}$ Department of Pathology, Cancer Institute, Imam Khomeini Hospital Complex, Tehran University of Medical Sciences, Tehran, Iran \\ ${ }^{2}$ Department of Pathology, Besat Hospital, Hamedan University of Medical Sciences, Hamedan, Iran \\ ${ }^{3}$ Department of Epidemiology, School of Public Health, Hamedan University of Medical Sciences, Hamedan, Iran \\ ${ }^{4}$ Department of Gynecology-Oncology, Alzahra Hospital, Tabriz University of Medical Sciences, Tabriz, Iran \\ "Corresponding author: Department of Pathology, Cancer Institute, Imam Khomeini Hospital Complex, Tehran University of Medical Sciences, Tehran, Iran. Email: \\ f-nili@sina.tums.ac.ir
}

Received 2019 October 29; Revised 2020 January 24; Accepted 2020 February 05.

\begin{abstract}
Background: Determining prognostic factors of endometrial cancer is important for the management of this disease.

Objectives: The aim of this study was to identify the association between tumor-infiltrating lymphocytes (TILs) and programmed cell death protein-1 (PD-1) expression in intra-tumoral inflammatory cells and clinicopathological features of endometrial carcinoma.

Methods: This observational study was conducted on 56 patients who underwent hysterectomy with diagnosis of endometrial carcinoma and available 5-year follow-up data from 2006 to 2013 in Imam Khomeini Hospital, Tehran, Iran. The TILs scoring was calculated based on the mean percentage of stroma occupied by mononuclear inflammatory cells at the invasive border of the tumor in $\mathrm{H} \& \mathrm{E}$ stained slides in 10 representative high-power fields. The mean number of CD3, CD8, and PD-1 positive lymphocytes in 10 high-power fields, as well as cyclo-oxygenase-2 (COX-2) expression, were assessed.

Results: TILs were significantly higher in endometrioid carcinoma compared to non-endometrioid carcinomas $(\mathrm{P}=0.001)$. There was no significant association between TILs percentage, myometrial invasion, lymphovascular invasion, and tumor grade $(\mathrm{P}>0.05)$. High TILs tumors showed better prognosis $(\mathrm{P}=0.046)$. On the immunohistochemical $(\mathrm{IHC})$ study, TILs were higher in endometrioid tumors compared to non-endometrioid tumors $(\mathrm{P}<0.05)$. The expression of PD-1 was higher in endometrioid tumors compared to non-endometrioid carcinomas $(\mathrm{P}=0.001)$. The COX-2 expression was not associated with prognosis and the other clinicopathologic features.

Conclusions: High TILs and PD-1 expression were associated with better prognosis in endometrial carcinoma. Endometrioid carcinomas had a higher TILs, CD3, CD8, and PD-1 lymphocytes. This study failed to identify a clinically significant cut off for these inflammatory biomarkers.
\end{abstract}

Keywords: Endometrial Carcinoma, Tumor Infiltrating Lymphocyte, Clinicopathologic Features, Prognosis

\section{Background}

Endometrial cancer is the sixth most common cancer in women worldwide $(1,2)$. The increasing trend in endometrial cancer is reported in several countries especially in regions with the rapid socioeconomic transition (1). This cancer is the most common malignancy in the female genital tract with more than 60,000 new cases and over 10,000 annual deaths (2). Based on 2-tiered classification by Bokhman, endometrial carcinoma is subcategorized as type I and II according to histopathological, clin- ical, and endocrinological features (3). Type I tumors are endometrioid carcinomas and their variants with a good prognosis in most cases. They usually occur in younger patients with an unopposed estrogen status (3). Type II tumors are composed of serous papillary and clear cell carcinomas with atrophic background, that occur in older patients usually with advanced disease at the time of presentation (3).

Clinical stage, histological subtype, FIGO grade, lymphovascular invasion and genomic alterations are the wellknown prognostic and predictive factors in endometrial 
carcinoma $(4,5)$. Recent studies have investigated several immunological biomarkers including tumor-infiltrating lymphocyte (TILs) as predictive tools in different cancers. Improved survival and response to treatment have been verified in different tumors in the majority of studies. However, there is limited data regarding the prognostic significance of TILs in endometrial carcinoma (6).

Cyclo-oxygenase-2 (COX-2), which converts arachidonic acid into prostaglandins and thromboxanes, plays a crucial role in the process of inflammation and tumor formation. Over-expression of COX-2 has an important role in tumor progression due to the enhancement of cell proliferation, inhibition of apoptosis, promotion of neoangiogenesis, stimulation of tumor COX-2 overmigration/invasion, and immune response suppression. The adverse prognostic significance of expression and the relationship with TILs in endometrial cancer has been investigated recently (7). Programmed cell death protein 1 (PD-1) is a member of the B7/cluster of differentiation (CD-28) family. It is an immune checkpoint receptor, which is expressed on T cells, B cells, natural killer cells, and monocytes. Programmed death ligand 1 (PD-L1) and programmed death-ligand 2 (PD-L2) are the ligands that interact with PD-1 to suppress T cell functions and induce tumor immune evasion. The PD-1/PD-L1 immune inhibitory pathway plays an important role in the ability of tumor cells to evade host immune response $(8,9)$.

\section{Objectives}

Accordingly, in this study, the association between TILs and PD1 expression in intra-tumoral inflammatory cells and clinicopathological features of endometrial carcinoma were assessed.

\section{Methods}

This observational comparative study was conducted on 56 consecutive patients who underwent hysterectomy and complete surgical staging due to endometrial carcinoma from 2006 to 2013 in Imam Khomeini Hospital, Tehran, Iran. The inclusion criteria were availability for follow up and the presence of optimal specimens for pathological assessment and immunohistochemical (IHC) study. Document papers of the patients from the Gyneco-oncology clinic in Imam Khomeini Hospital were reviewed for the patient's follow-up and slides from the Pathology Department were re-evaluated for pathologic parameters of the tumors and selecting the best paraffin block for IHC study. Patients with missing data or inappropriate pathology specimens were excluded. The study was approved by the Local Ethical Committee (code: IR.TUMS.IKHC.REC.1395.435) and the Helsinki Declaration was respected across the study.

The mean percentage of stroma occupied by mononuclear inflammatory cells at the invasive border of the tumor on $\mathrm{H} \& \mathrm{E}$ staining in 10 representative fields were used to identify the TILs score (Figure 1). In order to determine CD3 and CD8 counts, the number of CD3, CD8, and PD-1 positive lymphocytes in 10 representative high power fields $(40 \times$ microscopic field) at the invasive border of the tumor, intra-tumoral stroma, and within the tumor clusters were counted and the mean number for each area was calculated (Figure 2). The ratio of PD-1/CD3 lymphocytes in different areas was calculated and tumors with PD-1/CD3 percentage of more than $5 \%$ were considered as high.

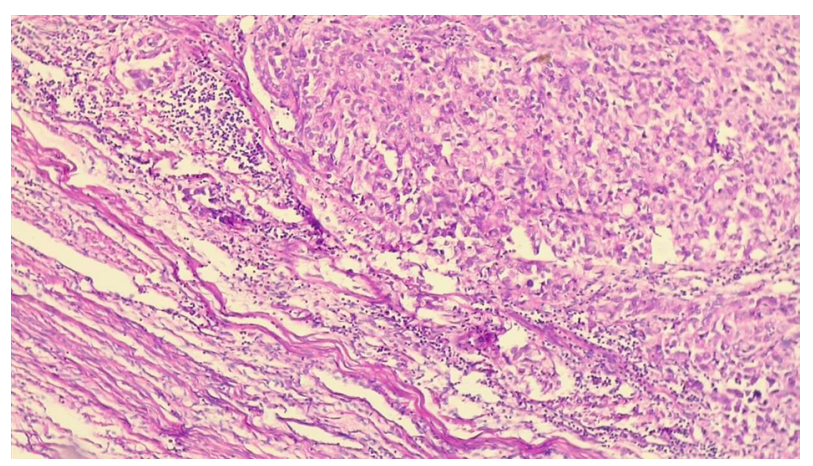

Figure 1. $H \&$ E stained sections $(100 \times)$ show the invasive border of the tumor, $5 \%$ $10 \%$ of the stroma is infiltrated by mononuclear inflammatory cells.

The COX-2 expression IHC scoring was performed based on the percentage of COX-2 positive tumor cells and intensity of staining. Sum of intensity score was categorized as follows; $1 \%$ - 5\%: (1) indicating weak expression, 5\% - 50\%; (2) indicating moderate expression and > 50\%; (3) indicating severe expression. Tumors with sum score $\geq 2$ were considered positive (Figure 3 ).

For IHC staining of all markers, including PD-1, CD3, CD8, and COX-2, the $4 \mu \mathrm{m}$ thick section was taken on charged slides and dried overnight at $60^{\circ} \mathrm{C}$. After deparaffinization and rehydration, heat-induced epitope retrieval was performed using the Master Diagnóstica EDTA buffer $(\mathrm{pH}=8)$ for $20 \mathrm{~min}$ at $95^{\circ} \mathrm{C}$. Upon completion, the slides were rinsed with distilled or deionized water followed by cooling at room temperature for $20 \mathrm{~min}$. Peroxidase solution was used, 10 minutes at room temperature for blocking endogenous peroxidase. After incubation for $10 \mathrm{~min}-$ utes with primary antibodies for CD3, CD8, COX-2, and PD1 (Master Diagnóstica; Spain), master polymer plus detection system (HRP) was used. Finally, counterstaining with haematoxylin and mounting of the slides were performed. 

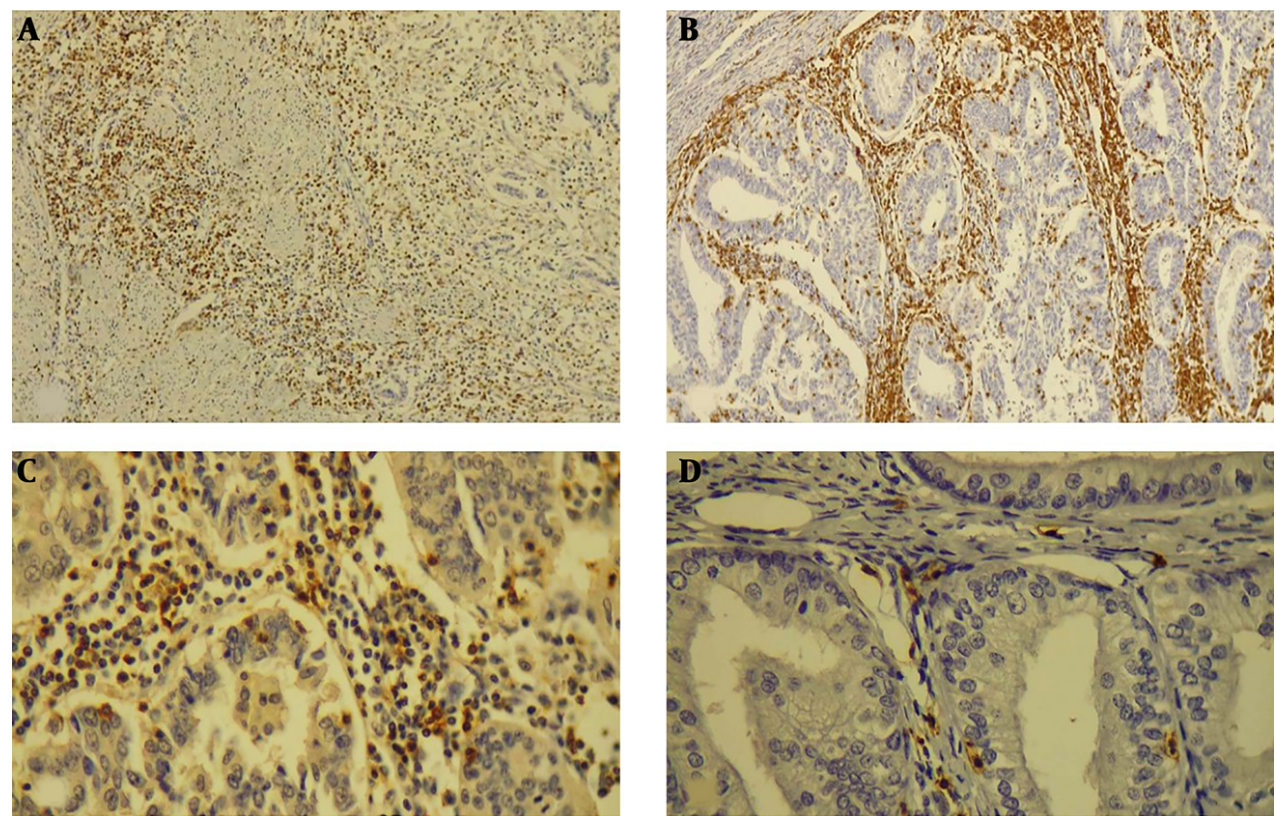

Figure 2. IHC study showing: A, the invasive border of tumor infiltrated by CD3 positive lymphocytes $(100 \times)$; B, invasive border and intratumoral areas with dense infiltration of CD3 positive T-cells (100X); C, infiltration of CD8 positive T cells in the tumoral stroma and intraepithelial region $(400 \times)$; $D$, infiltration of PD-1 positive $T$ cells in the tumoral stroma and intraepithelial region $(400 \times)$.
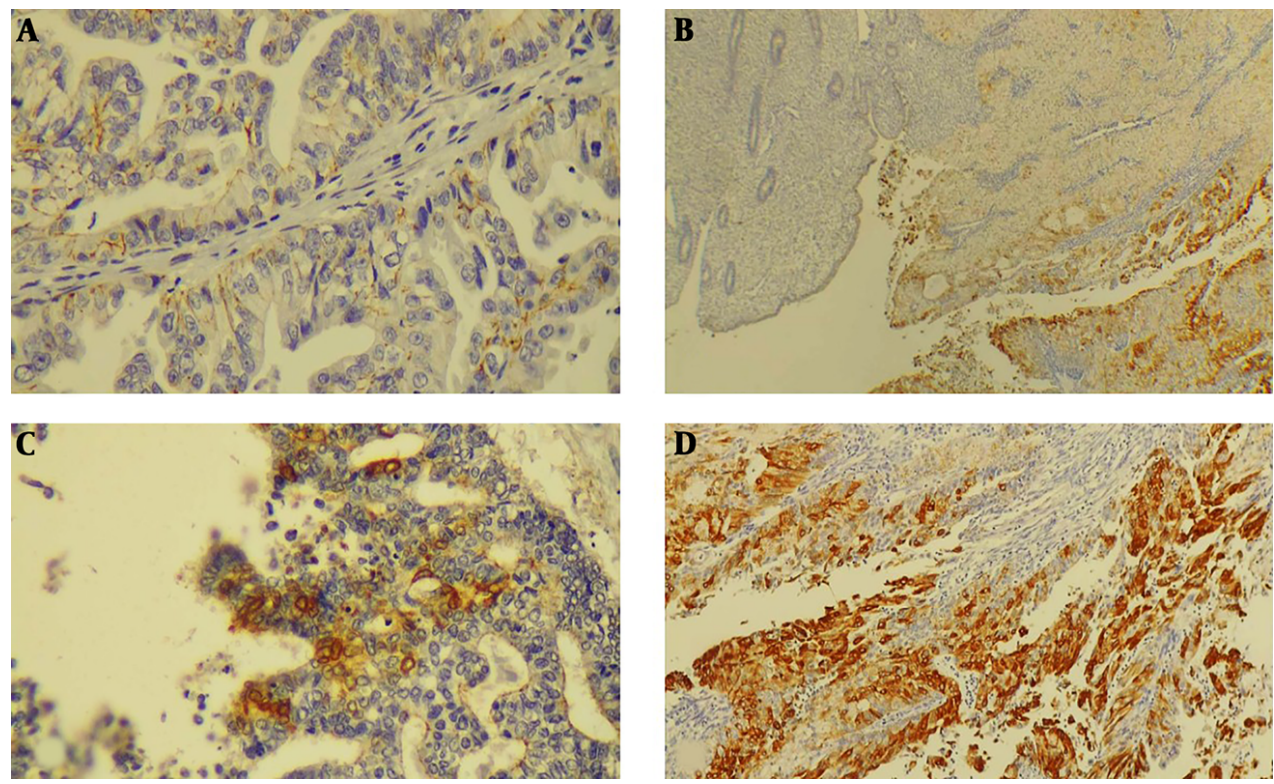

Figure 3. IHC study showing: A, weak staining with COX-2 $(400 \times)$; B, moderate staining with COX-2 in tumor cells and negative stain on normal endometrium $(100 \times)$; , moderate staining $(400 \times)$; D, severe staining.

Data analysis was performed using the statistical package for social sciences (SPSS) software version 22.0 (Chicago, Illinois, USA).The independent-sample $t$-test and the Pearson correlation coefficient were applied. The P val- ues less than 0.05 were considered as statistically significant. 


\section{Results}

A total of 56 patients were included in the study. Clinicopathologic features of the patients are summarized in Table 1. Most of the patients (91.1\%) were endometrioid subtype, well differentiated (39.3\%), and stage I (60.7\%). Finally, $85.8 \%$ of the patients were disease-free after 5 years. The others (14.9\%) presented with recurrence/metastatic disease and four patients died.

\begin{tabular}{|c|c|}
\hline Charachteristics & Values \\
\hline Age & $54.5 \pm 9.1(34-79)$ \\
\hline \multicolumn{2}{|l|}{ Histologic subtype } \\
\hline Endometrioid & $51(91.1)$ \\
\hline Non-Endometiriod & $5(8.9)$ \\
\hline Papillary serous & $4(7.1)$ \\
\hline Mixed & $1(1.78)$ \\
\hline Undifferentiated & $0(0)$ \\
\hline \multicolumn{2}{|l|}{ Histologic (FIGO) grade } \\
\hline Well (I) & $22(39.3)$ \\
\hline Moderate (II) & $19(33.9)$ \\
\hline Poor (III) & $15(26.8)$ \\
\hline \multicolumn{2}{|l|}{ Stage } \\
\hline I & $34(60)$ \\
\hline II & $9(16)$ \\
\hline III & $13(23)$ \\
\hline IV & 0 \\
\hline \multicolumn{2}{|l|}{ Outcome (5-year follow up) } \\
\hline Disease-free & $48(85)$ \\
\hline Recurrence/metastasis & $4(7.1)$ \\
\hline Death & $4(7.1)$ \\
\hline
\end{tabular}

The mean percentage of TILs was significantly higher in endometrioid carcinoma compared to nonendometrioid carcinomas $(P=0.001)$. There was no statistically significant difference in the mean number of the TILs between well, moderate, and poorly differentiated carcinomas $(\mathrm{P}>0.05)$. There was no statistically significant association between TILs percentage, myometrial invasion, and lymphovascular invasion ( $\mathrm{P}>0.05)$. Similarly, there was no statistically significant difference in TILs between different stages of endometrial carcinoma but TILs in patients with more aggressive behavior of the tumor, recurrence, and death were significantly lower than patients with disease-free 5-year follow-up $(\mathrm{P}=0.046)$ (Table 2).

Table 2. Relationship Between the Percentage of Stroma Occupied by Lymphocytes at the Invasive Border of the Tumor (TILs) and Clinicopathological Variables ${ }^{\mathrm{a}}$

\begin{tabular}{lcc}
\hline Clinicopathological Variables & Percentage of TILs & P Value \\
\hline Histologic type & & 0.01 \\
\hline Endometrioid & $25.49 \pm 23.8$ & \\
\hline Non-endometrioid & $10.6 \pm 4.9$ & \\
\hline FIGO grade & & \\
\hline I & $18.2 \pm 21$ & \\
\hline II & $31.9 \pm 28$ \\
\hline III & $23 \pm 15.5$ & \\
Stage & & \\
\hline I & $26.9 \pm 25.2$ & \\
\hline II & $22.7 \pm 23.1$ & \\
\hline III & $17.9 \pm 16.2$ & \\
\hline Outcome & & \\
\hline Disease-free & $25.2 \pm 24.2$ & \\
\hline Metastasis/recurrence/death & $15.2 \pm 9.2$ & \\
\hline
\end{tabular}

Abbreviations: FIGO, federation of obstetrics and gynecology; SD, standard deviation.

${ }^{\mathrm{a}}$ Values are expressed as mean $\pm \mathrm{SD}$.

On IHC study, the CD3 positive T lymphocytes at the invasive border of the tumor and intratumoral area, endometrioid tumors showed higher rates of infiltration of $\mathrm{T}$ lymphocytes compared to non-endometrioid carcinomas. Similar results were established for CD8 positive cytotoxic T lymphocytes.

Although the number of CD3 and CD8 positive lymphocytes were higher in low grade, early-stage, and tumors with better prognosis, the difference was not statistically significant (Table 3).

Intertumoral and stromal PD-1 positive lymphocytes were higher in endometrioid carcinomas. Tumors with better prognosis had more infiltration of PD1 positive cells but the difference between high and low grade tumors and stage were not statistically significant $(\mathrm{P}>0.05)$ (Table 4). Furthermore, there was no significant difference between patients with high and low PD1 positive cells and different histologic subtypes, grades and stages $(\mathrm{P}>0.05)$ (Table 4$)$.

Over-expression of COX-2 was not significantly associated with histologic subtype, grade, stage, and prognosis. There was no statistically significant difference between the number of TILs in COX-2 positive and negative tumors $(\mathrm{P}>0.05)$ (Table 5). 
Table 3. The Relationship Between Mean Number of CD3 and CD8 Positive Lymphocytes in 10 HPF, at Invasive Border of Tumor, Tumoral Stroma and Intra-Tumoral Area with Clinicopathological Features of Endometrial Carcinoma ${ }^{\mathrm{a}}$

\begin{tabular}{|c|c|c|c|c|c|c|}
\hline \multirow{2}{*}{ Clinicopathologic Variables } & \multicolumn{3}{|c|}{ CD3 (P Value) } & \multicolumn{3}{|c|}{ CD8 (P Value) } \\
\hline & Invasive Border & Intratumoral & Tumoral Stroma & Invasive Border & Intratumoral & Tumoral Stroma \\
\hline \multicolumn{7}{|l|}{ Histologic type } \\
\hline Endometrioid & $116 \pm 76$ & $16 \pm 17$ & $37 \pm 36$ & $80 \pm 64$ & $18.6 \pm 18.2$ & $21 \pm 17$ \\
\hline Non-endometrioid & $81 \pm 2$ & $1.4 \pm 1.3$ & $28 \pm 19$ & $56 \pm 9$ & $2.7 \pm 2.4$ & $22 \pm 15$ \\
\hline $\mathrm{P}$ & 0.03 & 0.000 & 0.025 & 0.025 & 0.000 & 0.9 \\
\hline \multicolumn{7}{|l|}{ FIGO grade } \\
\hline I & $134 \pm 80$ & $13 \pm 11$ & $34 \pm 29$ & $82 \pm 77$ & $15 \pm 6$ & $20 \pm 15$ \\
\hline II & $117 \pm 58$ & $11 \pm 10$ & $31 \pm 25$ & $78 \pm 58$ & $12 \pm 7$ & $19 \pm 17$ \\
\hline III & $109 \pm 51$ & $9 \pm 7$ & $30 \pm 23$ & $77 \pm 19$ & $11 \pm 4$ & $18 \pm 14$ \\
\hline $\mathrm{P}$ & 0.4 & 0.3 & 0.6 & 0.5 & 0.2 & 0.6 \\
\hline \multicolumn{7}{|l|}{ Stage } \\
\hline I & $132 \pm 78$ & $12 \pm 13$ & $33 \pm 25$ & $80 \pm 66$ & $13 \pm 8$ & $24 \pm 14$ \\
\hline II & $115 \pm 55$ & $10 \pm 8$ & $32 \pm 22$ & $79 \pm 56$ & $12 \pm 7$ & $20 \pm 15$ \\
\hline III & $111 \pm 54$ & $11 \pm 10$ & $31 \pm 20$ & $78 \pm 33$ & $10 \pm 6$ & $19 \pm 12$ \\
\hline $\mathrm{P}$ & 0.2 & 0.1 & 0.7 & 0.6 & 0.3 & 0.2 \\
\hline \multicolumn{7}{|l|}{ Outcome } \\
\hline Disease-free & $111 \pm 73$ & $17 \pm 15$ & $33 \pm 32$ & $79 \pm 60$ & $18 \pm 16$ & $22 \pm 19$ \\
\hline Metastasis/recurrence/death & $106 \pm 63$ & $12 \pm 9$ & $30 \pm 20$ & $76 \pm 54$ & $16 \pm 15$ & $21 \pm 18$ \\
\hline $\mathrm{P}$ & 0.2 & 0.3 & 0.5 & 0.4 & 0.6 & 0.8 \\
\hline
\end{tabular}

Abbreviations: FIGO, federation of obstetrics and gynecology; HPF, high power field; SD, standard deviation.

${ }^{\mathrm{a}}$ Values are expressed as mean $\pm \mathrm{SD}$.

\section{Discussion}

Endometrial carcinoma is a common gynecological cancer in developing countries. Numerous studies have been performed to identify the association between tumor related and general factors with the prognosis of endometrial carcinoma. TIL is an important factor in this era that indicates the immune response to the tumor. If such issues can be established, adjuvant immunotherapy would be beneficial and yield better outcomes in patients with high TILs. Robust evidence on the clinical significance of TILs in endometrial cancers is not established. In this study, the prognostic role of TILs at the invasive border of the tumor on $\mathrm{H} \& \mathrm{E}$ stained slides and specifically the number of CD3, CD8, and PD-1 positive lymphocytes in three regions, including the invasive border of the tumor, intra-tumoral stroma, and within the invasive tumor were assessed.

Evaluation of TILs was performed based on the recommendations by International TILs working group in breast cancer and other solid tumors (10). Based on this recommendation, the percentage of tumoral stroma at the invasive border of the tumor which was occupied by mononuclear inflammatory cells was assessed in this study. We found a good correlation between this method and accurate counting of $\mathrm{T}$ lymphocytes at the invasive border of the tumor on IHC study for CD3. The TILs were significantly higher in endometrioid versus non-endometrioid tumors. Patients with better prognosis had higher TILs compared to those with poorer outcomes. As this method is easier and cost-effective, it can be used in the routine practice of pathologists for reporting TILs in endometrial cancers.

To the best of our knowledge, there is no definite consensus on clinicopathologic features of endometrial carcinoma and immune-response (11). Silverberg et al. reported that high TILs are more common in high-grade endometrial cancers. They did not find a significant correlation between TILs and survival (12). In contrast, Deligdisch et al. found a higher number of TILs in low-grade carcinomas and suggested a better prognosis in these patients. Intraepithelial CD8 positive T cells were shown to be an independent predictor for prognosis in some studies (12-14). Kondratiev et al. (12) showed that the number of CD8+ lymphocytes was not correlated with tumor grade and stage, but it was significantly correlated with vascular invasion. Jung et al. (15) showed that the proportion of CD8 positive lympho- 
Table 4. The Relationship Between Number of PD1 Positive Lymphocytes and Percentage of PD1/CD3 Lymphocytes in the Tumor and Tumoral Stroma with Clinicopathological Features of the Patients

\begin{tabular}{|c|c|c|c|}
\hline $\begin{array}{l}\text { Clinicopathologic } \\
\text { Variables }\end{array}$ & $\begin{array}{l}\text { PD-1 Tumor }(P \\
\text { Value }\end{array}$ & $\begin{array}{l}\text { PD-1 Stroma }(P \\
\text { Value }\end{array}$ & $\begin{array}{c}\text { Percentage of } \\
\text { PD1 Positive T } \\
\text { Lymphocytes, } \\
\%\end{array}$ \\
\hline \multicolumn{4}{|l|}{$\begin{array}{l}\text { Histologic } \\
\text { type }\end{array}$} \\
\hline $\begin{array}{l}\text { En- } \\
\text { dometri- } \\
\text { oid }\end{array}$ & 5.04 & 13.01 & 61 \\
\hline $\begin{array}{l}\text { Non- } \\
\text { endometrioid }\end{array}$ & 0.4 & 3.2 & 2 \\
\hline $\mathrm{P}$ & 0.001 & 0.01 & 0.001 \\
\hline \multicolumn{4}{|l|}{ FIGO grade } \\
\hline I & 11.0 & 3.7 & 38 \\
\hline II, III & 14.6 & 6.0 & 35 \\
\hline $\mathrm{P}$ & 0.7 & 0.8 & 0.7 \\
\hline \multicolumn{4}{|l|}{ Stage } \\
\hline I & 6.7 & 14 & 45 \\
\hline II & 1.7 & 5.4 & 41 \\
\hline III & 1.0 & 10.0 & 41 \\
\hline $\mathrm{P}$ & 0.8 & 0.9 & 0.8 \\
\hline \multicolumn{4}{|l|}{ Outcome } \\
\hline $\begin{array}{l}\text { Disease- } \\
\text { free }\end{array}$ & 5.1 & 13.3 & 38 \\
\hline $\begin{array}{l}\text { Metasta- } \\
\text { sis/recurre }\end{array}$ & 1.7 & 4.9 & 37 \\
\hline $\mathrm{P}$ & 0.02 & 0.07 & 0.9 \\
\hline
\end{tabular}

Abbreviations: FIGO, federation of obstetrics and gynecology.

cytes was negatively correlated with tumor grade, myometrial invasion, and lymph node metastasis. In the present study, the number of CD3 and CD8 positive lymphocytes at the invasive border of the tumor and intraepithelial area were higher in endometrioid tumors in comparison with high-grade non-endometrioid carcinomas. Although TILs, $\mathrm{CD} 3$, and CD8 positive lymphocytes were higher in earlystage and low-grade tumors, we could not find a statistically significant difference. The small sample size in this study could be an influencing factor. de Jong et al. (13), in contrast with Kondratiev et al. (12), reported that the number of TILs was related to the type of tumor, which was in line with the findings of the present study.

Workel et al. (16) evaluated the prognostic role of CD103 positive intraepithelial lymphocytes (CD8+PD-1+T cell markers) and showed improved survival for high-risk endometrial cancers with a higher number of these lymphocyte markers. In the present study, the PD-1+/CD3+ lymphocytes ratio was significantly higher in patients with 5-

\begin{tabular}{|c|c|c|c|}
\hline $\begin{array}{l}\text { Clinicopathologic } \\
\text { Variables }\end{array}$ & COX-2 Positive & COX-2 Negative & PValue \\
\hline Histologic type & & & 0.27 \\
\hline Endometrioid & 39 & 9 & \\
\hline Non-endometrioid & 3 & 2 & \\
\hline FIGO grade & & & 0.62 \\
\hline I & 14 & 5 & \\
\hline II & 16 & 3 & \\
\hline III & 12 & 3 & \\
\hline Stage & & & 0.20 \\
\hline I & 24 & 7 & \\
\hline II & 8 & 1 & \\
\hline III & 10 & 3 & \\
\hline Outcome & & & 0.2 \\
\hline Disease-free & 37 & 8 & \\
\hline $\begin{array}{l}\text { Metasta- } \\
\text { sis/recurrence/death }\end{array}$ & 5 & 3 & \\
\hline TILs, \% & $22.7 \pm 22.1$ & $27.2 \% \pm 25$ & 0.53 \\
\hline
\end{tabular}

Abbreviations: FIGO, federation of obstetrics and gynecology; SD, standard deviation; TILs, tumor infiltrating lymphocytes.

${ }^{\mathrm{a}}$ Values are expressed as mean $\pm \mathrm{SD}$.

year disease-free survival and endometrioid carcinomas. But we could not find a clinically significant cut-off value.

The expression of COX-2 in tumor cells would result in the local extension of the tumor and would prevent the infiltration of lymphocytes into the tumor. The PGE2 induces COX-2 and inactivation of cytokines synthesis which would result in inhibition of CD8 positive T cells (17). Suemori et al. (17) found no association between COX-2 expression in endometrial MSI-negative cancers and prognosis but they identified a significant reverse association in MSI-positive cases. They found that MSI-positive tumors with lower COX-2 expression had a better prognosis (17). In our study, there was no association between COX-2 overexpression and intra-tumoral CD3 lymphocytes. No Clinicopathologic correlation between tumor grade, stage, subtype or prognosis, was also identified.

There is a scarcity of evidence regarding the prognostic value of TILs in endometrial carcinoma (11). Many studies have focused on TILs to predict MSI status. On the new classification of endometrial carcinomas, MSI tumors and POLE ultra-mutated subgroup have been found to have higher TILs and PDL-1 expression (5). Further studies with larger and higher assessment quality are required for the determination of TILs prognostic significance in endometrial carcinoma and its interactions with MSI and POLE sta- 
tus.

\subsection{Conclusions}

This study found that the higher number of tumorinfiltrating lymphocytes is associated with a better prognosis in patients with endometrial carcinoma. Furthermore, endometrioid carcinomas presented a higher count of TILs, CD3, CD8, and PD-1 positive T cells.

\section{Acknowledgments}

This study was supported by grant number: 95-02-3032034 in Tehran University of Medical Sciences.

\section{Footnotes}

Authors' Contribution: Study concept and design: Fatemeh Nili. Acquisition of data: Niusha Nobari. Analysis and interpretation: Fatemeh nili, Niusha Nobari and Jalal pooralajal Drafting of the manuscript: Nakisa Niknejad and Niusha Nobari. Critical revision of the manuscript for important intellectual content: Fatemeh Nili. Statistical analysis: Jalal Poorolajal. Administrative, technical, and material support: Issa Jahanzad. Study supervision: Fatemeh Nili.

Conflict of Interests: Nothing to be disclosed.

Ethical Approval: The study was approved by the local ethical committee (code: IR.TUMS.IKHC.REC.1395.435) and the Helsinki Declaration was respected across the study.

Funding/Support: The project has been supported by Tehran University of Medical Sciences.

\section{References}

1. Lortet-Tieulent J, Ferlay J, Bray F, Jemal A. International patterns and trends in endometrial cancer incidence, 1978-2013. J Natl Cancer Inst. 2018;110(4):354-61. doi:10.1093/jnci/djx214. [PubMed: 29045681].

2. Siegel RL, Miller KD, Jemal A. Cancer statistics, 2018. CA Cancer J Clin. 2018;68(1):7-30. doi: 10.3322/caac.21442. [PubMed: 29313949].

3. Lax SF. Pathology of endometrial carcinoma. Adv Exp Med Biol. 2017;943:75-96. doi: 10.1007/978-3-319-43139-0_3. [PubMed: 27910065].

4. Sorbe B. Predictive and prognostic factors in definition of risk groups in endometrial carcinoma. ISRN Obstet Gynecol. 2012;2012:325790. doi: 10.5402/2012/325790. [PubMed: 23209924]. [PubMed Central: PMC3504391].

5. Cancer Genome Atlas Research N, Kandoth C, Schultz N, Cherniack $\mathrm{AD}, \mathrm{Akbani} \mathrm{R}$, Liu Y, et al. Integrated genomic characterization of endometrial carcinoma. Nature. 2013;497(7447):67-73. doi: 10.1038/nature12113. [PubMed: 23636398]. [PubMed Central: PMC3704730].
6. Jia Q, Yang Y, Wan Y. Tumor-infiltrating memory T-lymphocytes for prognostic prediction in cancer patients: A meta-analysis. Int J Clin Exp Med. 2015;8(2):1803-13. [PubMed: 25932108]. [PubMed Central: PMC4402755].

7. Telliez A, Furman C, Pommery N, Henichart JP. Mechanisms leading to COX-2 expression and COX-2 induced tumorigenesis: topical therapeutic strategies targeting COX-2 expression and activity. Anticancer Agents Med Chem. 2006;6(3):187-208. doi:10.2174/187152006776930891. [PubMed: 16712448].

8. Bregar A, Deshpande A, Grange C, Zi T, Stall J, Hirsch H, et al. Characterization of immune regulatory molecules B7-H4 and PD-L1 in low and high grade endometrial tumors. Gynecol Oncol. 2017;145(3):44652. doi: 10.1016/j.ygyno.2017.03.006. [PubMed: 28347512].

9. Mo Z, Liu J, Zhang Q, Chen Z, Mei J, Liu L, et al. Expression of PD1, PD-L1 and PD-L2 is associated with differentiation status and histological type of endometrial cancer. Oncol Lett. 2016;12(2):944-50. doi: 10.3892/ol.2016.4744. [PubMed: 27446374]. [PubMed Central: PMC4950473]

10. Salgado R, Denkert C, Demaria S, Sirtaine N, Klauschen F, Pruneri G, et al. The evaluation of tumor-infiltrating lymphocytes (TILs) in breast cancer: Recommendations by an international TILs working group 2014. Ann Oncol. 2015;26(2):259-71. doi: 10.1093/annonc/mdu450. [PubMed: 25214542]. [PubMed Central: PMC6267863].

11. Hendry S, Salgado R, Gevaert T, Russell PA, John T, Thapa B, et al. Assessing tumor-infiltrating lymphocytes in solid tumors: A practical review for pathologists and proposal for a standardized method from the International Immunooncology Biomarkers Working Group: Part 1: Assessing the host immune response, TILs in invasive breast carcinoma and ductal carcinoma in situ, metastatic tumor deposits and areas for further research. Adv Anat Pathol. 2017;24(5):235-51. doi: 10.1097/PAP.0000000000000162. [PubMed: 28777142]. [PubMed Central: PMC5564448].

12. Kondratiev S, Sabo E, Yakirevich E, Lavie O, Resnick MB. Intratumoral CD8+ T lymphocytes as a prognostic factor of survival in endometrial carcinoma. Clin Cancer Res. 2004;10(13):4450-6. doi: 10.1158/10780432.CCR-0732-3. [PubMed: 15240536].

13. de Jong RA, Leffers N, Boezen HM, ten Hoor KA, van der Zee AG, Hollema $\mathrm{H}$, et al. Presence of tumor-infiltrating lymphocytes is an independent prognostic factor in type I and II endometrial cancer. Gynecol Oncol. 2009;114(1):105-10. doi: 10.1016/j.ygyno.2009.03.022. [PubMed: 19411095]

14. Cermakova P, Melichar B, Tomsova M, Zoul Z, Kalabova H, Spacek J, et al. Prognostic significance of CD3+ tumor-infiltrating lymphocytes in patients with endometrial carcinoma. Anticancer Res. 2014;34(10):5555-61. [PubMed: 25275055].

15. Jung IK, Kim SS, Suh DS, Kim KH, Lee CH, Yoon MS. Tumor-infiltration of T-lymphocytes is inversely correlated with clinicopathologic factors in endometrial adenocarcinoma. Obstet Gynecol Sci. 2014;57(4):266-73. doi: 10.5468/ogs.2014.57.4.266. [PubMed: 25105099]. [PubMed Central: PMC4124087].

16. Workel HH, Komdeur FL, Wouters MC, Plat A, Klip HG, Eggink FA, et al. CD103 defines intraepithelial CD8+ PD1+ tumour-infiltrating lymphocytes of prognostic significance in endometrial adenocarcinoma. Eur J Cancer. 2016;60:1-11. doi: 10.1016/j.ejca.2016.02.026. [PubMed: 27038842].

17. Suemori T, Susumu N, Iwata T, Banno K, Yamagami W, Hirasawa A, et al. Intratumoral CD8+ lymphocyte infiltration as a prognostic factor and its relationship with cyclooxygenase 2 expression and microsatellite instability in endometrial cancer. Int J Gynecol Cancer. 2015;25(7):1165-72. doi: 10.1097/IGC.0000000000000482. [PubMed: 26111272]. 